\title{
Spatial Accessibility to Sports Facilities in Dongguan, China: A Multi-Preference Gaussian Two- Step Floating Catchment Area Method
}

\section{Tong Xiao}

Sun Yat-Sen University

\section{Tengfei Ding}

Sun Yat-Sen University

\section{Xiaoke Zhang}

Sun Yat-Sen University

\section{Zhuolin Tao}

Beijing Normal University

Ye Liu ( $\square$ liuye25@mail.sysu.edu.cn )

Sun Yat-Sen University https://orcid.org/0000-0003-2511-5413

\section{Research}

Keywords: sports facilities, equity, spatial accessibility, multi-preference Gaussian two-step floating catchment area method, Dongguan

Posted Date: June 28th, 2021

DOl: https://doi.org/10.21203/rs.3.rs-585644/v1

License: (c) (1) This work is licensed under a Creative Commons Attribution 4.0 International License. Read Full License

Version of Record: A version of this preprint was published at Applied Spatial Analysis and Policy on February 10th, 2022. See the published version at https://doi.org/10.1007/s12061-022-09436-4. 
6 Tong Xiao

Tengfei Ding

Zhuolin Tao

\title{
Spatial Accessibility to Sports Facilities in Dongguan, China: A Multi-Preference
}

\section{Gaussian Two-step Floating Catchment Area Method}

\author{
Tong Xiao ${ }^{1,2}$, Tengfei Ding ${ }^{1 \dagger}$, Xiaoke Zhang ${ }^{1 *}$, Zhuolin $\mathrm{Tao}^{3}$, Ye Liu ${ }^{1,2 *}$
}

School of Geography and Planning, Sun Yat-Sen University, Xingang Xi Road, Guangzhou 510275,

China; Guangdong Key Laboratory for Urbanization and Geo-simulation, Sun Yat-Sen University,

Xingang Xi Road, Guangzhou 510275, China; Email: xiaot9@mail2.sysu.edu.cn

School of Geography and Planning, Sun Yat-Sen University, Xingang Xi Road, Guangzhou 510275, China; Email: dingtf@mail2.sysu.edu.cn

\section{Xiaoke Zhang}

School of Geography and Planning, Sun Yat-Sen University, Xingang Xi Road, Guangzhou 510275,

China; Email: zhangxk9@mail2.sysu.edu.cn

Faculty of Geographical Science, Beijing Normal University, No.19, XinJieKouWai St., Haidian District, Beijing 100875, China; Email: taozhuolin@bnu.edu.cn

Ye Liu* (Corresponding author)

School of Geography and Planning, Sun Yat-Sen University, Xingang Xi Road, Guangzhou 510275, China; Guangdong Key Laboratory for Urbanization and Geo-simulation, Sun Yat-Sen University, Xingang Xi Road, Guangzhou 510275, China; Email: liuye25@mail.sysu.edu.cn

\footnotetext{
$\dagger$ These authors contributed equally.

* These authors contributed equally.
} 


\section{Abstract}

2 Background: Equitable access to sports services has drawn attention from policymakers and

3 planners in China as people's health has been placed at the centre of the country's policy-

4 making machinery. However, existing approaches to measure the spatial accessibility of sports

5 facilities tend to ignore the heterogeneity in potential users' demands and facility preferences,

6 thereby causing a bias in the measurement of accessibility.

7 Methods: To accurately measure the spatial accessibility of sports facilities, this paper 8 proposes a multi-preference Gaussian two-step floating catchment area (MG2SFCA) method,

9 taking into account different travel modes, catchment sizes, and facility preferences among 10 different age groups. Empirically, using high-resolution gridded data on actual population

11 distribution, we adopted the MG2SFCA method in examining the spatial accessibility of sports

12 facilities among children, working-age population, and elderly population under walking,

13 cycling, and driving modes in Dongguan, China. Further, a comparison was made between the accessibility results measured by the MG2SFCA method and the Gaussian 2SFCA method.

15 Results: The results indicated a significant spatial disparity in the accessibility to sports 16 facilities, with better accessibility in the north and poorer accessibility in the south. Children 17 have the best access to sports facilities, followed by working-age and elderly populations. In the comparison among different transport modes, the accessibility distribution of sports

19 facilities in walking mode showed the greatest spatial variation, while accessibility in driving mode was the most balanced. The spatial variation of accessibility scores of MG2SFCA was more significant than that of Gaussian 2SFCA.

22 Conclusions: The MG2SFCA method is superior to the traditional Gaussian 2SFCA method 
1 because the former can capture disadvantaged people's actual needs for sports facilities more

2 accurately than the latter. Introducing demographic and socioeconomic characteristics of

3 individuals into spatial accessibility measurement has a vital impact on the accurate reflection

4 of accessibility inequality.

6 Keywords: sports facilities, equity, spatial accessibility, multi-preference Gaussian two-step

7 floating catchment area method, Dongguan

9 List of abbreviations:

10 2SFCA: two-step floating catchment area; MG2SFCA: multi-preference Gaussian two-step floating 11 catchment area; E2SFCA: enhanced two-step floating catchment area; CHNS: China Health and 12 Nutrition Survey.

\section{Introduction}

16 Emerging evidence suggests that regular physical activity has a beneficial impact on physical

17 and mental health, including increased positive effect, less loneliness, and reduced rates of obesity, diabetes and cardiovascular disease (Bourke et al., 2021; Gyasi et al., 2020; Hunter

19 and Reddy, 2013; Senaratne et al., 2021; White et al., 2017; Zulyniak et al., 2020). With

20 increasing emphasis on health and well-being, the promotion of physical activity has aroused

21 intense attention from the government, the public, and researchers. In this regard, a plethora of studies have reported that the availability and accessibility of sports and recreational services 
1 play a prominent role in physical activity attainment (Halonen et al., 2015; Heath et al., 2012;

2 Humpel et al., 2002; Liu et al., 2019). In 2020, the World Health Organization (WHO)

3 recommended at least 600 metabolic equivalent minutes of physical activity each week.

4 However, at present, approximately a quarter of adults and three-quarters of adolescents around

5 the world fail to reach this recommendation, which is partially attributable to poor planning

6 and design of sports and recreational facilities (WHO, 2018). Although the quantity and quality

7 of facilities in most high-income regions have improved significantly over the past few decades,

8 there is still a shortage of facilities in many low-income to middle-income regions. Furthermore,

9 facilities are unequally distributed in most areas, especially in developing countries.

11 In recent years, China's sports industry has boomed, with a per capita sports field area of 1.86

$12 \mathrm{~m}^{2}$ by the end of 2019 , but is still far smaller than that of developed countries (National Bureau

13 of Statistics of China, 2019). Furthermore, this figure varies greatly among the eastern, central,

14 and western parts of China. The 14th Five-Year Plan of China (2021) points out that the

15 protection of public health should be given priority in the strategic position of development,

16 with the goal of building China into a strong country in sports. Special attention should be

17 given to the vulnerable groups to improve the population service system, with the focus on

'one old and one young'. Moreover, the construction of all-age-friendly cities requires more

19 attention to the needs of children and the elderly to enhance their sense of happiness and gain.

20 Considering the unique hierarchical characteristics of sports facilities in China, as well as the

21 current problem of unequal spatial distribution, it is necessary to propose a new approach targeted to examine the spatial accessibility of sports facilities among different age groups, 
1 with particular focus on children and the elderly. An accurate assessment of accessibility helps

2 to efficiently identify underserved areas and populations, thus further providing evidence-based

3 guidelines for urban planners and policymakers on how to allocate sports facilities reasonably

4 to improve equalisation of public services. A reasonable layout of sports facilities makes

5 accessibility easier, thus increasing enthusiasm for physical activities and subsequently

6 contributing to better physical and mental health.

8 Uneven geographic distributions of supply and demand lead to varied spatial accessibility to 9 services (Wang and Luo, 2005). Spatial accessibility refers to the ease, potential, or 10 opportunities for interaction between supply and demand points in the transport network, which 11 highlights spatial separation (e.g. geographic location and distance) that can act as either a 12 barrier or a facilitator (Hansen, 1959; Morris et al., 1979; Pirie, 1979). Previous studies have 13 proposed several approaches to evaluate accessibility to public services for different purposes and understandings of the concept. Provider-to-population ratio and distance measures (i.e. the

15 Euclidean/road network/travel time distance to the closet service) are the simplest and most 16 widely used indicators (Neutens, 2015; Pirie, 1979). Nonetheless, the former does not consider 17 the geographical barrier between supply and demand, while the latter ignores the functional 18 diversity and scale differences of services. Therefore, slightly more complex geographic 19 information system (GIS)-based approaches are employed to analyse the relationship between 20 supply and demand from a spatial perspective, which can be summarised as three types of 21 models: resistance models, cumulative opportunity measures, and gravity-type measures 22 (Hansen, 1959; Kwan, 1998; Neutens, 2015). Among these, the two-step floating catchment 
1 area (2SFCA) model is relatively straightforward to interpret and easy to implement, and it has

2 become one of the most popular methods for examining geographical access to opportunities

3 and services. As a special case of the gravity-based model, it enables consideration of

4 geographic barriers and interactions between suppliers and demanders, as well as distance

5 decay effects (Luo and Wang, 2003). Moreover, it innovatively employs a dichotomous

6 technique to address distance attenuation from consumers to providers based on a predefined

7 catchment area, irrespective of the constraints of administrative boundaries, thus encompassing

8 cross-boundary availability of services (Dai, 2010; Fransen et al., 2015; Tao and Cheng, 2016;

9 Yang et al., 2006). With increasing research, various continuous distance decay functions, 10 catchment area sizes, supply and demand competition, and multiple travel modes have been 11 proposed to advance 2SFCA metrics (Tao and Cheng, 2016).

13 Several studies have investigated the spatial accessibility of sports facilities to provide insight into proper allocation and design (Billaudeau et al., 2011; Cereijo et al., 2019; Cutumisu and Spence, 2012; Higgs et al., 2015; Karusisi et al., 2013; Shrestha et al., 2019). However, most researchers have used simple indicators, such as distance to the nearest facility and the number

17 of facilities within a predefined circular buffer around the dwelling or in a specific administrative unit (Billaudeau et al., 2011; Cereijo et al., 2019; Halonen et al., 2015; Karusisi et al., 2013; Shrestha et al., 2019), with only some studies using 2SFCA models (Cutumisu and Spence, 2012; Higgs et al., 2015; Langford et al., 2018). Cutumisu and Spence (2012) adopted an enhanced 2SFCA (E2SFCA) method to evaluate objective accessibility to sports fields at the census block level and further compared the impacts of objectively or subjectively assessed 
1 accessibility on physical activity levels among adults in Edmonton, Canada. Similarly, Higgs

2 et al. (2015) applied the same model to examine the inequality of access to sports facilities

3 among regions with different socioeconomic statuses in Wales. Moreover, Langford et al. (2018)

4 created an add-in tool in ArcGIS to facilitate the calculation process of E2SFCA for spatial

5 accessibility to sports facilities. However, these studies followed the methods proposed by

6 previous studies and did not specifically consider the characteristics of sports facilities in

7 evaluating accessibility (Luo and Qi, 2009). Additionally, these studies have taken census tracts

8 or postal code areas as the minimum unit of accessibility analysis, failing to accurately

9 determine the geographical location of demand points. Moreover, people of different

10 characteristics, such as age, gender, ethnicity, education level and income, tend to have different

11 preferences and levels of participation in physical activities, which affect the choice of sports

12 facilities (Bélanger et al., 2011; Farrell and Shields, 2002). The 1997 health survey of England

13 has indicated that males are more likely to participate in cycling, football or rugby, gym, racket

14 sports, and running, while females have higher participation rates in aerobics, dancing, and

15 swimming (Farrell and Shields, 2002). Although some studies compared the variations in

16 access to sports facilities in regions with different socioeconomic conditions, they did not go

17 into detail at the population level and distinguished the demand population according to

18 demographic characteristics and socioeconomic attributes.

20 To advance the field of measuring the spatial accessibility of sports facilities, we aim to propose

21 a new, improved model, the multi-preference Gaussian two-step floating catchment area

22 (MG2SFCA) method based on the original 2SFCA, allowing for a more accurate and rational 
1 measurement. To this end, we make methodological enhancements in terms of data accuracy,

2 individual preference, catchment area, and travel mode. Specifically, fine-grained age-specific

3 georeferenced population data were used and transformed into $100 \mathrm{~m} \times 100 \mathrm{~m}$ gridded data to

4 determine the demand points. This also allowed us to capture individual attributes and group

5 the total population by age; thus, the accessibility for different age groups could be assessed

6 separately. In particular, based on the national sample survey data, the preference indexes of

7 different age groups for the usage of sports facilities were collected and included in the

8 MG2SFCA as the weight of service supply. In addition, considering the hierarchical

9 characteristics of sports facilities in China, we allocated catchment areas of diverse sizes to

10 facilities of different levels in accordance with their service scope. Multiple modes of transport

11 were also incorporated into the accessibility evaluation. The proposed method is employed to

12 estimate spatial access to sports facilities for three age groups (children, working-age adults,

13 and the elderly) under three separate travel modes (walking, cycling, and driving) in Dongguan,

14 China. This new approach not only uniquely focuses on the needs of children and the elderly,

15 but also considers the different preferences of different age groups. Therefore, the application

16 of the MG2SFCA method provides a more realistic and reliable evaluation of spatial access to

17 sports facilities, which is conducive to accurately identifying the unreasonable space layout of services. On this basis, evidence-based implications can be provided for the planning and

19 redevelopment of public services to maximise the use efficiency and promote the equalisation

20 of public services.

\section{Review of 2SFCA metrics}


1 Radke and $\mathrm{Mu}$ (2000) first proposed a spatial decomposition method to quantify spatial

2 accessibility, which was subsequently modified by Luo and Wang (2003) and named as 2SFCA.

3 The basic 2SFCA calculates the provider-to-population ratio within the catchment area centred

4 at a provider's location, and then aggregates the ratios of all providers within the catchment

5 centred on a population point to evaluate spatial accessibility.

7 Nonetheless, the traditional 2SFCA method has some deficiencies; thus, a number of

8 improvements have been proposed (Table 1). First, the application of the dichotomous

9 technique leads to consistent access to services within the catchment area, while services

10 outside the catchment are completely inaccessible (Tao and Cheng, 2016). Accordingly, several

11 distance decay functions have been proposed to delineate the differences in geographical

12 accessibility within the catchment. For instance, Luo and Qi (2009) presented an E2SFCA to

13 deal with the problem by applying different weighting values calculated from the Gaussian

14 function to three previously divided discrete travel time zones. Although the E2SFCA identifies

15 service shortage areas more explicitly, it has no uniform standard for segmenting the catchment,

16 as well as assigning equal access within each segment. Then, the Gaussian 2SFCA was

17 developed to estimate health care access, which employed a Gaussian function to continuously capture the distance decay effect of accessibility within a catchment (Dai, 2010; 2011). Dai and

19 Wang (2011) introduced a kernel density function into the original 2SFCA to continuously

20 delineate distance decay within the catchment. In addition, many other distance decay functions

21 and metrics have been used to improve the original 2SFCA, such as linear, exponential, and inverse power (Neutens, 2015; Tao and Cheng, 2016). Furthermore, to deal with the uncertainty 
1 caused by the selection of the impedance coefficient in a distance decay function, Lin et al.

2 (2018) introduced a spatial access ratio into the model, which was determined by comparing

3 the accessibility score ratio of a given place to the average accessibility score of the study area.

4

5

Table 1. Various improved forms of 2SFCA

\begin{tabular}{|c|c|c|c|}
\hline Aspect & Method & Inventors & Advantages \\
\hline Distanc & Enhanced 2SFCA (E2SFCA) & Luo and Qi (2009) & Apply different weights to multiple travel time zones \\
\hline \multirow[t]{3}{*}{ e decay } & Gaussian 2SFCA & Dai $(2010 ; 2011)$ & Use a Gaussian function as distance decay function \\
\hline & Kernel density 2SFCA & Dai and Wang (2011) & Use a kernel density function as distance decay function \\
\hline & (KD2SFCA) & & \\
\hline \multirow{9}{*}{$\begin{array}{l}\text { Catchm } \\
\text { ent size }\end{array}$} & Variable 2SFCA (V2SFCA) & Luo and Whippo (2012) & Dynamically increase catchment sizes according to whether the \\
\hline & & & minimum value \\
\hline & $\begin{array}{l}\text { 2SFCA with dynamic catchment } \\
\text { sizes }\end{array}$ & $\begin{array}{l}\text { McGrail and Humphreys } \\
\qquad \text { (2014) }\end{array}$ & $\begin{array}{l}\text { Set a five-level dynamic catchment size based on population's } \\
\text { remoteness, and add a three-level catchment sub-type between } \\
\text { remoteness levels }\end{array}$ \\
\hline & (Nearest-neighbour modified & Jamtsho et al. (2015) & Assume that people seek services from the nearest facilities \\
\hline & 2SFCA) NN-M2SFCA & & \\
\hline & Multiple catchment sizes & Tao et al. (2014) & Set variable catchment sizes according to the scales of services \\
\hline & 2SFCA (MC2SFCA) & & \\
\hline & $\begin{array}{l}\text { Gravity-based variable 2SFCA } \\
\qquad \text { (GV2SFCA) }\end{array}$ & Tao et al. (2020a) & $\begin{array}{l}\text { Set variable catchment sizes for different regions and various levels } \\
\text { of services }\end{array}$ \\
\hline & Hierarchical 2SFCA (H2SFCA) & Tao et al. (2020b) & Set variable catchment sizes according to the hierarchies of services \\
\hline Travel & Multi-mode 2SFCA & Mao and Nekorchuk (2013) & Divide population into several subgroups according to travel modes \\
\hline \multirow[t]{2}{*}{ mode } & / & Langford et al. (2016) & Assign a dedicated transport network for each travel mode \\
\hline & / & Xing et al. (2018) & $\begin{array}{l}\text { Set different travel modes for the population in different locations } \\
\text { according to the distance between supply points and demand points }\end{array}$ \\
\hline \multirow[t]{4}{*}{ Other } & Optimised 2SFCA & Ngui and Apparicio (2011) & Take actual users as demanders \\
\hline & $\begin{array}{l}\text { Three-step floating catchment } \\
\text { area method (3SFCA) }\end{array}$ & Wan et al. (2012) & Incorporate a spatial impedance-based competition scheme \\
\hline & Modified 2SFCA (M2SFCA) & Delamater (2013) & Allow suboptimal configurations of services \\
\hline & Huff 2SFCA & Luo (2014) & Introduce the Huff model to delineate facility selection behaviour \\
\hline
\end{tabular}

6

7 Second, it is not reasonable to establish a single catchment area for different facilities on 
1 account of their distinctions in category, scale, level, etc. To address this issue, Luo and Whippo

2 (2012) proposed a variable 2SFCA that incrementally increased the catchment area until the

3 minimum population threshold and minimum per capita service threshold were reached.

4 Additionally, McGrail and Humphreys (2014) implemented a five-level dynamic catchment

5 size in accordance with population density and created smoother transition catchment sizes at

6 remoteness-level boundaries. While the modified method provides a more accurate assessment

7 of spatial accessibility, it is more difficult to operate in practice. Meanwhile, given the assumption that people tend to choose facilities closer to them rather than seeking all services

9 within the catchment, Jamtsho et al. (2015) utilised a nearest-neighbour (NN) method to set catchment sizes. As different sizes of facilities have different spatial service scopes, larger

11 facilities were designated with larger catchment areas in the multiple catchment sizes 2SFCA

12 (MC2SFCA) (Tao et al., 2014). However, Tao et al. (2020a; 2020b) later modified this view and argued that the catchment area should be determined according to the hierarchies of services rather than their sizes, and the variable catchment areas were assigned to different regions and different levels of services in the gravity-based variable 2SFCA (GV2SFCA) and hierarchical 2SFCA (H2SFCA).

18 Third, considering the diversity of travel modes, Mao and Nekorchuk (2013) suggested a multi-

19 mode 2SFCA method in which the population at each facility location was divided into $n$ subgroups by transport modes, allowing for more realistic estimations of spatial accessibility. Langford et al. (2016) further improved the assignment of a dedicated transport network for each travel mode. In addition, Xing et al. (2018) set different travel modes in different locations 
1 according to the distance between the supply and demand points. Owing to the dynamic nature

2 of human mobility, GPS trajectory data (e.g. mobile phone tracking data and taxi trajectories)

3 have also been applied to detect travel behaviour (Chen et al., 2020a, 2020b; Xia et al., 2019).

4 Instead of assuming that all facilities within the travel time threshold were available, Wang et

5 al. (2020) examined hospital accessibility by incorporating taxi trip records to determine

6 whether there was an actual interaction between the population and healthcare facilities. Chen

7 et al. (2020b) divided school trips into two types: Home-School and Home-School-Work, and

8 applied the commuter-based 2SFCA proposed by Fransen et al. (2015) that introduced trip-

9 chaining behaviour in evaluating accessibility.

11 Some advanced 2SFCA metrics concerning other aspects have also been proposed. To

12 minimise demand overestimation, Ngui and Apparicio (2011) considered actual users as

13 demanders instead of the total population within the catchment (optimised 2SFCA), while Wan et al. (2012) assigned a spatial impedance-based competition weight to each pair of population and service sites on the basis of a three-step FCA (3SFCA). Since 3SFCA has ignored the influence of supply capacity on selection behaviour, Luo (2014) adopted the Huff model to compute selection probability of available services (Huff 2SFCA). Meanwhile, Delamater (2013) developed a modified 2SFCA (M2SFCA) method that calculated the specific supply ratios for each supply-demand pair, considering both relative and absolute distances from population to services. Subal et al. (2021) implemented a modified Huff 3SFCA method based on the M2SFCA and Huff 2SFCA to consider the absolute distance and avoid demand overestimation. Moreover, unlike previous studies that mostly considered area as an indicator 
1 of provision, Xing et al. (2018) incorporated the varying sizes and functions of parks into

2 accessibility evaluation. Other studies have also investigated the change in accessibility over

3 time and its association with socioeconomic status from a spatiotemporally dynamic

4 perspective (Li et al., 2019; Hu et al., 2020).

6 Overall, the existing advanced forms of 2SFCA for measuring spatial accessibility have

7 improved to varying degrees in terms of distance attenuation, catchment area, supply and

8 demand competition, and travel mode. However, these methods have mostly been applied to

9 healthcare facilities, residential care facilities, and green spaces, and the use of advanced

10 2SFCA in measuring the accessibility of sports facilities is rare. Few studies have considered

11 the impact of individual heterogeneity and facility characteristics on the decision-making

12 process, as well as the importance of accurate geocoding of demanders. Considering the above

13 deficiencies, with fine-grained population data and detailed facility data, we used age as a basis

14 for population grouping to assess the accessibility of different groups, as well as set variable

15 catchment sizes according to the service scopes corresponding to the level of sports facilities.

16 Methodologically, we extracted the preference indexes on the usage of facilities of different

17 age groups from nationally represented survey data and calculated the weight of service supply

18 accordingly. At the same time, three travel modes (walking, cycling, and driving) were

19 considered to measure accessibility under different transport scenarios.

21 3. Data and methods

\subsection{Study area}


1 The case study area is Dongguan City, located on the east bank of the Pearl River Estuary in

2 south-central Guangdong, China. There are four subdistricts and 28 towns in the study area,

3 with 350 village committees and 242 resident committees under the jurisdiction of these

4 subdistricts and towns (Fig. 1). The city covered a total area of approximately 2,460 $\mathrm{km}^{2}$ and,

5 by the end of 2019 , had a permanent population of more than 8.4645 million, among which

6 migrants accounted for over 70\% (Dongguan Bureau of Statistics and National Bureau of

7 Statistics of China, 2020). As a famous sports city, the per capita sports field area (including

8 all kinds of sports facilities) reaches $3.19 \mathrm{~m}^{2}$, ranking at the forefront. However, there is still a

9 structural imbalance and low public openness in the construction of sports facilities in

10 Dongguan, and the quality and layout must also be improved. Thus, enabling equitable access

11 to sports facilities has become a pressing issue in Dongguan.

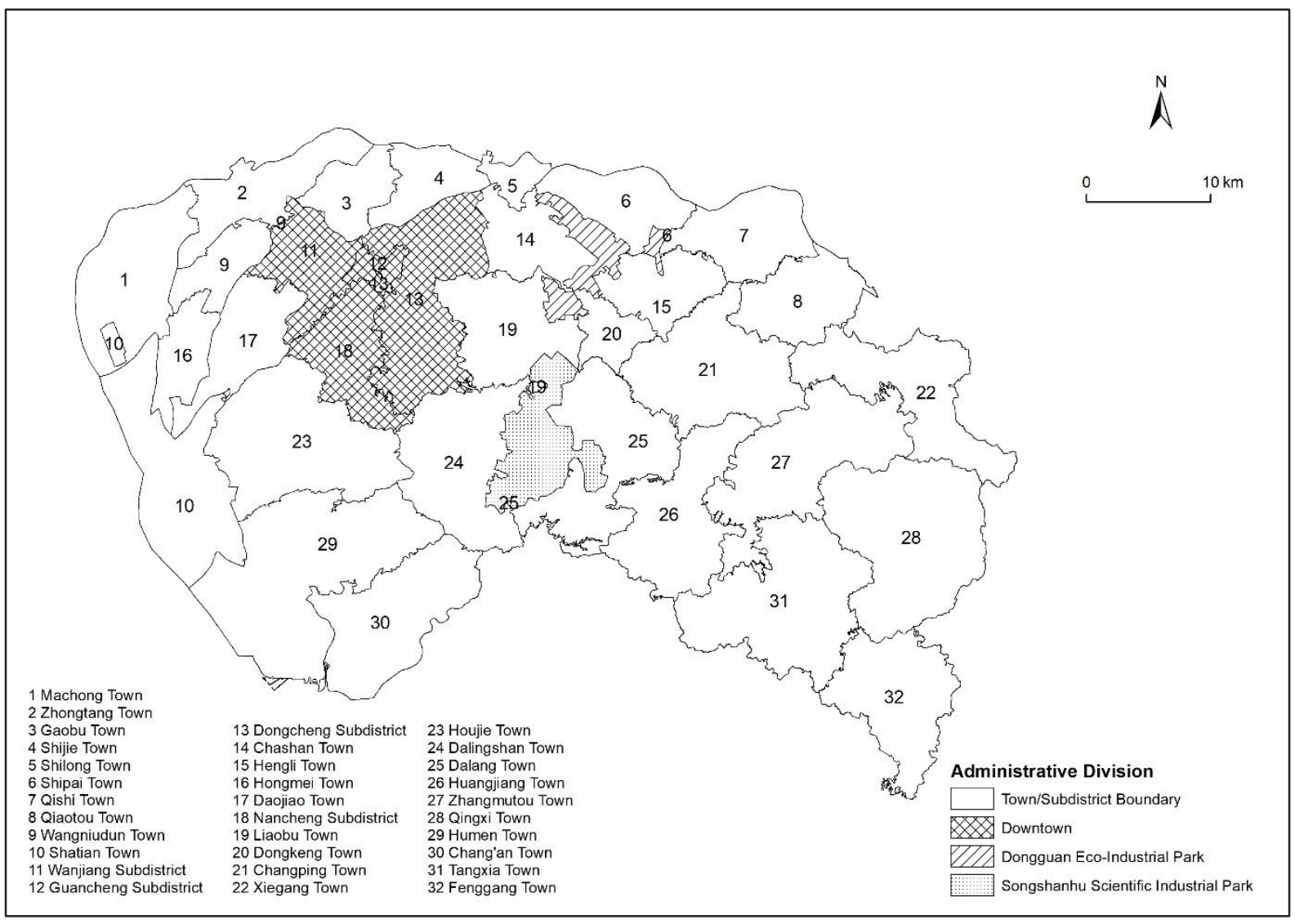

Figure 1. Administrative division of Dongguan City 
2 Three primary data sources were used in this study: sports facilities, population distribution,

3 and road networks. All datasets were obtained from the Dongguan Planning Bureau and the

4 Dongguan Public Security Bureau.

$6 \quad$ 3.2.1 Sports facilities

7 In the present study, sports facilities refer to various types of facilities and fields that can be

8 used for carrying out physical exercise, including privately or publicly owned, free or paid,

9 indoor or outdoor sports facilities, such as stadiums, swimming pools, ball game venues, fitness

10 routes and specific training sites. Golf courses and other sports venues catering only to a very

11 small number of users were not included. The exhaustive list of facilities has provided

12 information on the name, type, address, grade, building area, and site area of the facilities. All

13 facilities generated geographic coordinates according to the address and service sites. After

14 excluding facilities that did not meet the requirements, the final service dataset included 4,076

15 facilities with attributes and coordinates ${ }^{\S}$. Referring to relevant planning in China, the facilities

16 were divided based on their scale and service scope into three levels: municipal (129), town

17 (346), and primary (3,601) (Fig. 2).

§The final service dataset included 1,583 basketball courts, 169 all-people fitness paths, 98 swimming pools, 68 fitness rooms, 67 track and field grounds, 40 badminton courts, 30 football fields, 28 sports park, 205 other independent sports facilities, and 1,788 integrated sports facilities. 


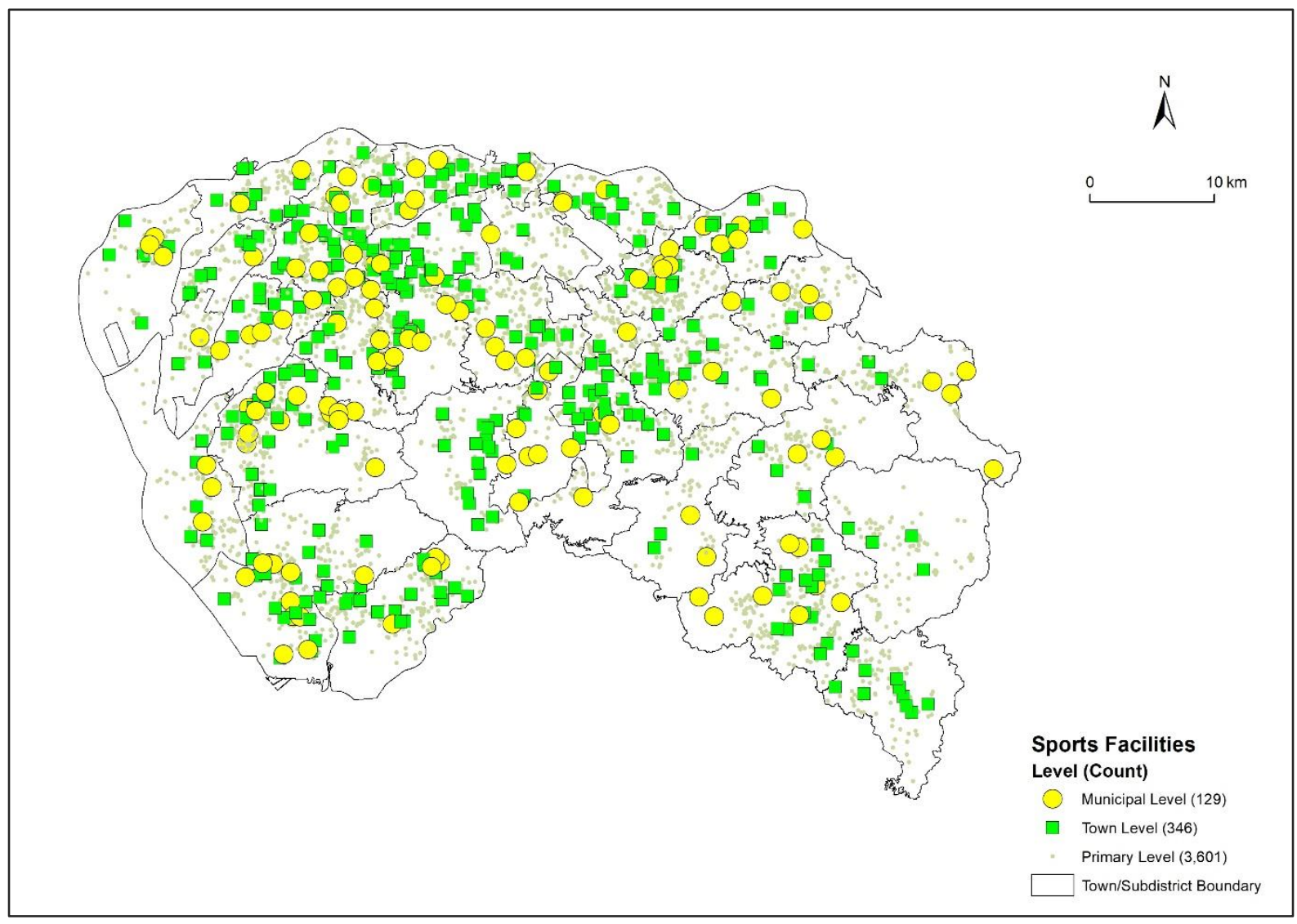

Figure 2. Spatial distribution of sports facilities

\subsubsection{Actual population}

5 The population data were derived from the basic information database of 'One standard and

6 Three actualities' collected by the Dongguan Public Security Bureau from 2018 to 2019. 'One

7 standard' refers to the standard address database, and 'Three actualities' refers to the real

8 population (including registered, transient, and overseas population), real houses and real units.

10 Unlike previous studies that used census data (Bell et al., 2013), the present study used

11 population data aggregated from individuals with georeferenced residential addresses. The total

12 population $(9,364,168)$ in Dongguan was classified into three age groups: children aged 0-14

13 years $(454,187)$, working-age population aged $15-59$ years $(8,475,595)$, and elderly population 
1 aged $\geq 60$ years $(430,343)$ (Fig. 3). To facilitate the analysis, a grid operation was carried out

2 on the base map of the Dongguan administrative division in ArcGIS 10.5, with 248,141 grid

3 cells of $100 \mathrm{~m} \times 100 \mathrm{~m}$ size in total. Then, the total population of each grid cell was calculated.

4 Assuming an even distribution in each grid cell, the cells with population attributes were

5 converted into point data and taken as demand sites. After excluding pseudo-demand sites

6 without any population, 66,071 demand sites with a non-zero population were included in the

$7 \quad$ spatial accessibility analysis.

8

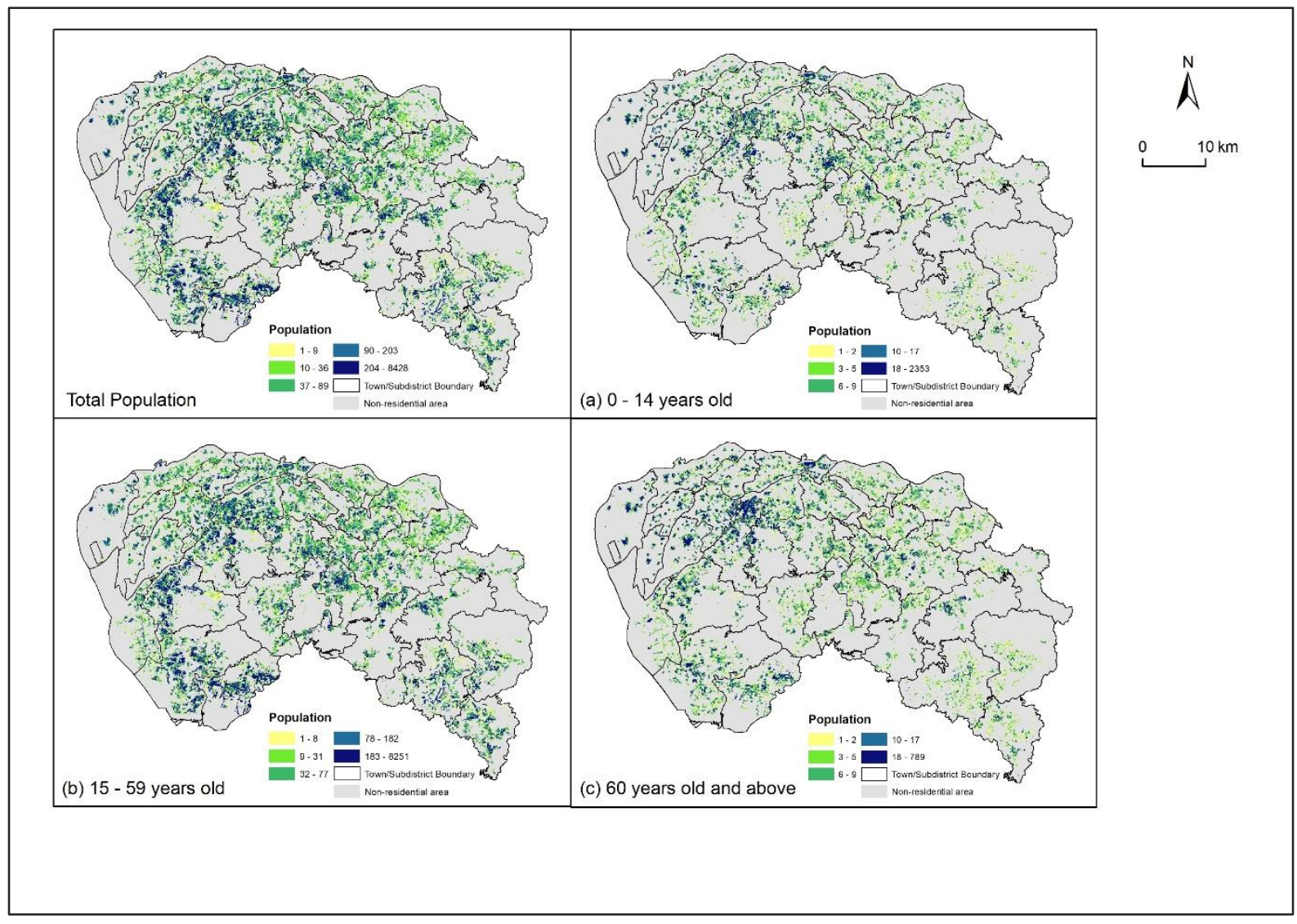

Figure 3. Spatial distribution of population

\subsubsection{Road network}

13 The road network included all urban roads, excluding highways and tracks. Each road and 
1 street contained information on name, length, width, road level, and speed class. In light of the existing research, combined with the Code for transport planning on urban road (GB 50220-

3 95) and the actual driving speed in Dongguan, the driving speed for different roads was set as

4 follows: $60 \mathrm{~km} / \mathrm{h}$ for expressway, $40 \mathrm{~km} / \mathrm{h}$ for arterial road, $30 \mathrm{~km} / \mathrm{h}$ for secondary trunk road,

$520 \mathrm{~km} / \mathrm{h}$ for branch road, and $10 \mathrm{~km} / \mathrm{h}$ for other lower level roads. In reference to the average

6 daily travel speed, the riding speed and walking speed of all roads were set at $250 \mathrm{~m} / \mathrm{min}$ and

$7 \quad 80 \mathrm{~m} / \mathrm{min}$, respectively.

\section{$9 \quad 3.3$ Methods}

10 An MG2SFCA was adopted to evaluate the spatial accessibility and equity of sports facilities

11 in Dongguan. Because sports facilities are a type of non-emergency leisure and entertainment

12 services, and their functional differences do not have a significant impact on decision-making,

13 the Gaussian function is more suitable than the basic 2SFCA in delineating the variation trend

14 of distance attenuation of sports facilities in supply-demand interactions within a catchment.

15 The Gaussian 2SFCA method proposed by Dai $(2010 ; 2011)$ was implemented in two steps.

16 The first step is to search all demand points $(k)$ within the catchment area $d_{0}$ centring on

17 each supply point $j$. The demand population is weighted by the Gaussian function $(G)$, and then added as potential users to calculate the supply to-demand ratio $\left(R_{j}\right)$. The second step is

19 to search all services $(l)$ within the catchment area $d_{0}$ centring on each population point $i$ 20 and add all supply to-demand ratios $\left(R_{l}\right)$ weighted by the Gaussian function $(G)$. The formula 21 is set as follows: 
1

2

$$
A_{i}=\sum_{l \in\left\{d_{i l} \leq d_{0}\right\}} R_{l} G\left(d_{i l}, d_{0}\right)=\sum_{l \in\left\{d_{i l} \leq d_{0}\right\}} \frac{s_{j} G\left(d_{i l}, d_{0}\right)}{\sum_{k \in\left\{d_{k j} \leq d_{0}\right\}} P_{k} G\left(d_{k j}, d_{0}\right)}
$$

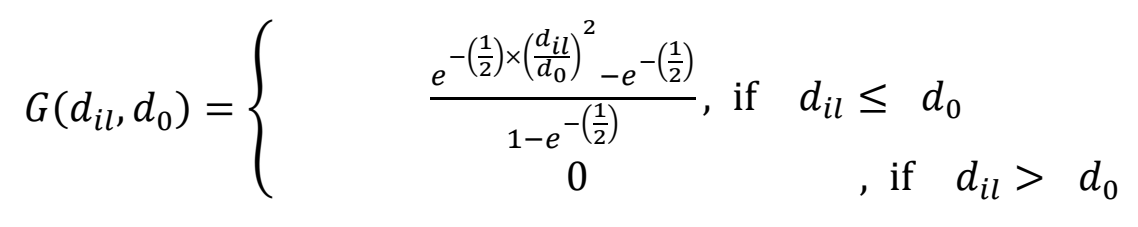

5 where $P_{k}$ is the population falling within the catchment $\left(d_{k j} \leq d_{0}\right), S_{j}$ is the supply capacity 6 at location $j, d_{k j} / d_{i l}$ is the travel time between $k / i$ and $j / l, G$ is the friction of distance

7 based on the Gaussian function that continuously accounts for the distance decay of access to

8 the service, and $A_{i}$ is the accessibility for population point $i$.

10 Based on the analysis and summary of various forms of existing 2SFCA methods for spatial 11 accessibility analysis, a modified model with optimisation in variable catchment sizes based on the level of facilities, multiple transport modes, and preference heterogeneity among

13 different age groups was proposed. Specifically, we improved the original Gaussian 2SFCA method by replacing the single catchment size for all facilities with multi-catchment sizes. In this study, different levels of sports facilities with disparities in capacity were assigned to distinct catchment sizes. The catchment area was quantified by the amount of travel time

17 (average in minutes) taken from the location of the demand population to the service site.

18 According to the Standard for Urban Public Service Facilities Planning (GB50442), the travel

19 time thresholds for municipal, township, and primary sports facilities were set at $30 \mathrm{~min}, 20$

$20 \mathrm{~min}$, and $10 \mathrm{~min}$, respectively. Considering the diversity of transport modes, the spatial accessibility to sports facilities for people in walking mode, cycling mode and driving mode 
3 Additionally, considering that different age groups may have different preferences for sports

4 and have different demands for a particular type of facility, a weighting factor of facility supply

5 based on the preference index was incorporated into the modified model. Using physical

6 activity and fitness data from the 2008 Health Survey for England, Bélanger et al. (2011)

7 confirmed that age was a major determinant of the time spent on specific types of physical

8 activity and behaviour related to using sports services. Operationally, we divided the population

9 into three age groups: children (age 0-14 years), working-age population (age 15-59 years),

10 and elderly population (age $\geq 60$ years). We then computed statistics on the preference of each

11 age group for various types of sports facilities based on the 2015 China Health and Nutrition

12 Survey (CHNS 2015). The CHNS is a nationally representative longitudinal study that began

13 in 1989, covering the eastern, central, and western parts of China, and was designed to

investigate a series of economic, sociological, demographic, nutrition, and health questions (Popkin et al., 2010). In 2015, a total of 20,914 individuals of all ages from 12 provinces (Guangxi, Guizhou, Heilongjiang, Henan, Hubei, Hunan, Jiangsu, Liaoning, Shaanxi, Shandong, Yunnan, and Zhejiang) and three autonomous cities/districts (Beijing, Chongqing, and Shanghai) were recruited through a multistage random cluster process. After excluding samples with missing relevant information, 16,622 respondents were included in the sports preference analysis, including 2,348 children, 9,600 working-age adults, and 4,674 elderly. Although Dongguan was not included in the survey, the sample is representative because of the coverage of regions in China with different geographical locations and economic development 
1 levels, and Dongguan has a large number of migrants from these regions. In CHNS 2015, the

2 preference index for a certain sports facility in the three age groups was quantified as the

3 proportion of people who were engaged in particular physical activities in the total age group

4 population (Table 2 )

5

6

Table 2. The proportion of people using different types of sports facilities in their age group

Type of sports facility
Group A: Children
Group B: Working-

age population
Group C: Elderly

population
Type 1: Comprehensive gymnasium

Type 2: Martial arts hall, gymnastics hall, dancing hall

Type 3: Track and field ground, swimming pool

Type 4: Fitness route

Type 5: Soccer field, basketball court, tennis court

Type 6: Badminton court, volleyball court

Type 7: Other
$33.83 \%$

$18.51 \%$

$21.41 \%$

$1.63 \%$

$10.87 \%$

$7.12 \%$

$8.87 \%$
$13.21 \%$

$3.07 \%$

$3.78 \%$

$4.01 \%$

$2.09 \%$

$1.85 \%$

$3.38 \%$
$13.36 \%$

$2.45 \%$

$1.93 \%$

$6.08 \%$

$0.11 \%$

$0.33 \%$

$4.15 \%$

7

8 Overall, we measured the spatial accessibility to sports facilities of each of the three age groups

9 under each of the three modes of transport in Dongguan, thereby performing nine rounds of

10 accessibility analysis with the same approach. Classifying the children in driving mode (riding

11 their parents' or other adults' cars) as an example, the MG2SFCA is implemented in the

12 following two steps:

14 Step 1: For each service $j$ of Type $n$ ( $n$ ranges from 1 to 7 ), the catchment was defined as a travel time threshold $\left(d_{0}\right)$ based on facility level (municipal level: $30 \mathrm{~min}$, town level: $20 \mathrm{~min}$, 
1 and primary level: $10 \mathrm{~min}$ ). We searched all demand sites $(k)$ within the corresponding

2 catchment area $\left(d_{k j} \leq d_{0}\right)$ and added up the population aged 0-14 years as children who are

3 potential users of service $j$. Then, the supply to-demand ratio $\left(R_{A j}\right)$ at service site $j$ was

4 calculated by dividing the supply capacity $\left(S_{j}\right)$ by the number of children users $\left(P_{A k}\right)$ :

$$
R_{A j}=\frac{s_{j} * W_{A j}}{\sum_{k \in\left\{d_{k j} \leq d_{0}\right\}} G\left(d_{k j}, d_{0}\right) P_{A k}}
$$

(3)

$$
W_{A j}=\frac{\sum_{k \in\left\{d_{k j} \leq d_{0}\right\}} G\left(d_{k j}, d_{0}\right) P_{A k} U_{A n}}{\sum_{k \in\left\{d_{k j} \leq d_{0}\right\}} G\left(d_{k j}, d_{0}\right)\left(P_{A k} U_{A n}+P_{B k} U_{B n}+P_{C k} U_{C n}\right)}
$$

where $P_{A k}, P_{B k}$ and $P_{C k}$ are the total population of children, working-age population, and elderly population groups falling within the catchment $\left(d_{k j} \leq d_{0}\right)$ of service $j$, respectively; $d_{k j}$ is the travel time from demand site $k$ to service site $j ; U_{A n} / U_{B n} / U_{C n}$ refers to the preference index of children/working-age population/elderly population for sports facilities of

14 Type $n$, which is computed by dividing the age group population using sports facilities of Type $n$ by the total corresponding age group population (as shown in Table 2); $W_{A j}$ is the weight

16 that represents the expected supply for the children group at service site $j$ (Type $n$ ), which is

17 calculated in accordance with preference indexes of three age groups; $W_{A j}+W_{B j}+W_{C j}=1$;

$18 S_{j}$ is the capacity of service $j$, which is quantified as site area $\left(\mathrm{m}^{2}\right)$; and $G\left(d_{k j}, d_{0}\right)$ is the

19 friction of distance between service site $j$ and demand site $k$.

21 Step 2: For each demand point $(i)$, we searched all services $(l)$ within the corresponding 
1 catchment area $\left(d_{i l} \leq d_{0}\right)$ and added up the supply-to-demand ratio $\left(R_{A l}\right)$. Then, the total

2 accessibility $A_{A i}$ for demand population $i$ for all sports facilities was obtained:

$$
A_{A i}=\sum_{i \in\left\{d_{i l} \leq d_{0}\right\}} G\left(d_{i l}, d_{0}\right) R_{A l}
$$

where $R_{A l}$ is the supply-to-demand ratio at service site $l$ falling within the catchment of

7 demand site $i\left(d_{i l} \leq d_{0}\right), d_{i l}$ is the travel time from demand site $i$ to service site $l$; and $8 G\left(d_{i l}, d_{0}\right)$ is the friction of distance between demand site $i$ and service site $l$. The output unit

9 is per capita area (square metres per person). The 13th Five-Year Plan for Sports Development 10 of China (2016) has set a target of $1.8 \mathrm{~m}^{2}$ per capita by 2020. According to the People's

11 Government of Guangdong Province (2020), the per capita sports area should reach $2.6 \mathrm{~m}^{2}$ by 2025 and $2.9 \mathrm{~m}^{2}$ by 2035, while the Dongguan Municipal People's Government (2020) requires that the city's per capita sports area should reach $3.2 \mathrm{~m}^{2}$ by 2025 . Therefore, we define all target values as the break values of the accessibility interval. All of the above analyses were conducted in ArcGIS 10.5, and Python 3.8.3.

\section{4. Results}

\subsection{Accessibility for three age groups under walking mode}

19 The spatial accessibility of different age groups under walking, cycling, and driving modes is

20 shown in Fig. 4. There was a substantial spatial variation in the overall accessibility scores under the walking mode. For children, relatively low accessibility scores are distributed in the central (Dongcheng and Chashan), southeast (Tangxia and Fenggang), and southwest (Humen 
1 and Chang'an) areas. For the working-age group, the high scores are scattered, mainly

2 concentrated in the northwest (Zhongtang, Wangniudun, Hongmei, Gaobu, Daojiao, and

3 Wanjiang), while the low scores are widespread in the middle (Dongcheng, Chashan, and

4 Liaobu) and south (Humen, Chang'an, Tangxia, Houjie, Qingxi, and Fenggang) regions. For

5 the elderly, the accessibility scores in most areas are low, except for a few northern areas (Qishi,

6 Daojiao, Qiaotou, and Wangniudun). With increase in age, the number of areas with high

$7 \quad$ accessibility are decreased.

8

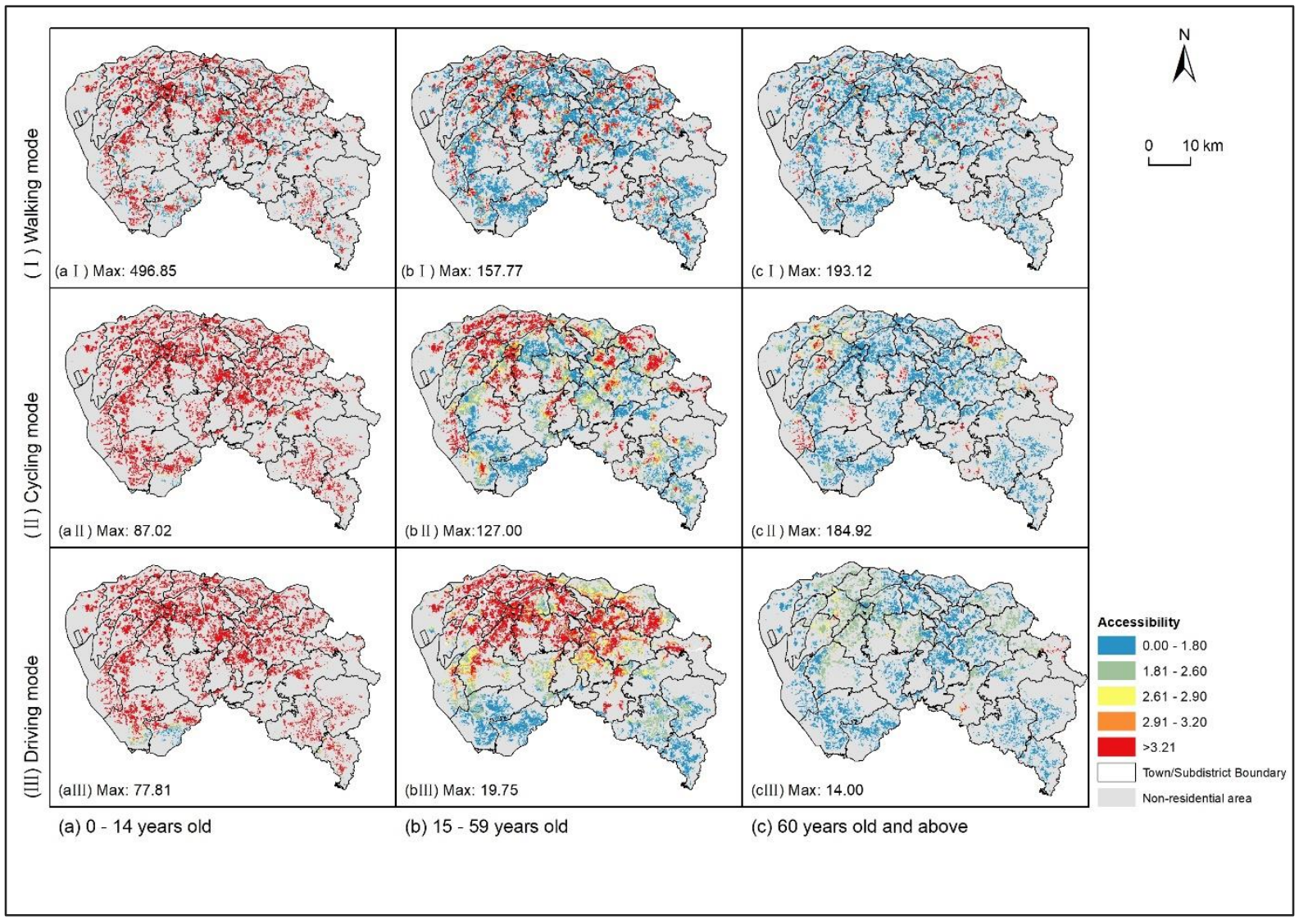

Figure 4. Spatial accessibility of sports facilities for three age groups

12 Relevant statistics of the spatial accessibility scores are presented in Table 3 . With an average

13 score of 10.23, children have a high score in accessibility, meeting Dongguan's 2025 target 
$1 \quad\left(3.2 \mathrm{~m}^{2}\right.$ per capita). The average scores for the working-age and elderly groups are much lower

2 (2.37 and 1.26 , respectively). Only $23.12 \%$ of the areas are underserved for children $\left(\leq 1.8 \mathrm{~m}^{2}\right.$

3 per capita), while the proportion of underserved areas for the working-age and elderly groups

4 is approximately three times $(62.65 \%)$ and four times $(81.64 \%)$ that of children, respectively.

5 Moreover, the variances show that the spatial accessibility gap narrows as age increases.

6

7

Table 3. The statistical characteristics of spatial accessibility to sports facilities

\begin{tabular}{|c|c|c|c|c|c|c|c|c|c|}
\hline \multirow{2}{*}{ MG2SFCA score } & \multicolumn{3}{|c|}{ Walking mode } & \multicolumn{3}{|c|}{ Cycling mode } & \multicolumn{3}{|c|}{ Driving mode } \\
\hline & $0-14$ & $15-59$ & $\geqslant 60$ & $0-14$ & $15-59$ & $\geqslant 60$ & $0-14$ & $15-59$ & $\geqslant 60$ \\
\hline Mean value & 10.23 & 2.37 & 1.26 & 11.77 & 2.64 & 1.46 & 13.19 & 2.82 & 1.50 \\
\hline Median value & 5.75 & 1.13 & 0.42 & 10.78 & 2.29 & 1.08 & 13.62 & 2.94 & 1.54 \\
\hline Maximum value & 496.85 & 157.77 & 193.12 & 87.02 & 127.00 & 184.92 & 77.81 & 19.75 & 14.00 \\
\hline Variance & 289.37 & 22.94 & 14.10 & 40.76 & 5.61 & 9.17 & 29.12 & 1.44 & 0.59 \\
\hline $0.00-1.80$ (Proportion) & $23.12 \%$ & $62.65 \%$ & $81.64 \%$ & $0.70 \%$ & $33.10 \%$ & $77.27 \%$ & $1.41 \%$ & $21.53 \%$ & $62.42 \%$ \\
\hline $1.81-2.60$ (Proportion) & $7.59 \%$ & $10.69 \%$ & $6.90 \%$ & $0.96 \%$ & $26.84 \%$ & $12.08 \%$ & $1.61 \%$ & $17.82 \%$ & $34.29 \%$ \\
\hline 2.61-2.90 (Proportion) & $2.44 \%$ & $3.16 \%$ & $1.60 \%$ & $0.46 \%$ & $7.45 \%$ & $2.41 \%$ & $0.53 \%$ & $9.12 \%$ & $2.06 \%$ \\
\hline 2.91-3.20 (Proportion) & $2.42 \%$ & $2.84 \%$ & $1.23 \%$ & $0.62 \%$ & $5.56 \%$ & $1.87 \%$ & $0.46 \%$ & $10.10 \%$ & $0.59 \%$ \\
\hline 3.21-7.00 (Proportion) & $19.96 \%$ & $14.40 \%$ & $5.70 \%$ & $20.49 \%$ & $25.44 \%$ & $5.15 \%$ & $10.43 \%$ & $41.18 \%$ & $0.43 \%$ \\
\hline$>7.00$ (Proportion) & $44.47 \%$ & $6.27 \%$ & $2.93 \%$ & $76.77 \%$ & $1.61 \%$ & $1.23 \%$ & $85.56 \%$ & $0.25 \%$ & $0.21 \%$ \\
\hline
\end{tabular}

8

94.2 Accessibility for three age groups under cycling mode

10 In the cycling mode, the overall score of accessibility was slightly higher than that in the

11 walking mode. For children, high accessibility scores cover almost the entire city, with $97.26 \%$ 
1 of the grids with scores $\geq 3.2 \mathrm{~m}^{2}$ per capita. For the working-age group, high scores

2 agglomerate in the north areas (Zhongtang, Wangniudun, Hongmei, Gaobu, Daojiao, Qishi,

3 Qiaotou, Xiegang, and Wanjiang), while low scores are in towns near the southern boundary

4 (Humen, Chang'an, Tangxia, Houjie, Qingxi, and Fenggang). Most areas scored low on

5 accessibility for elderly, with high scores in a few areas (Qiaotou, Shatian, Wangniudun, 6 Daojiao, and Wanjiang).

7

8 The differentiation degree in spatial accessibility was the highest in the children group and

9 lowest in the working-age group. Notably, the accessibility in the downtown area is high in the

10 working-age group but low in the elderly group, which can be partly attributed to more elderly

11 people and relatively fewer working-age adults in the downtown area. Similarly, as the

12 working-age group is mainly located in the south (Houjie, Qingxi, Humen, and Chang'an) and

13 middle (Guancheng and Dongcheng) areas, their accessibility is low despite having a large 14 number of sports facilities.

\subsection{Accessibility for three age groups under driving mode}

17 The spatial distribution of accessibility under the driving mode shares some features with those of the previous two modes. For children, the range of high-value areas is further expanded, but

19 some areas in the southwest still have low scores. For the working-age group, accessibility scores in the north are generally higher than those in the south. For the elderly group, accessibility scores are mostly low except in downtown and nearby areas. 
1 When driving to sports facilities, all age groups have more equalised values of spatial

2 accessibility, with variances of 29.12 (0-14 years old), 1.44 (15-59 years old), and 0.59 (60

3 years old and above). The mean and median values of accessibility for the same age group were

4 very similar, with a difference within 0.5 . For the elderly, the accessibility value in $62.42 \%$ of

5 the area is still $<1.8 \mathrm{~m}^{2}$ per capita, compared with only $1.41 \%$ for children and $21.53 \%$ for

6 working-age adults.

7

8

9 The results measured using Gaussian 2SFCA and MG2SFCA were compared. Regardless of the method used, the overall accessibility of sports facilities in Dongguan is at a high level under cycling and driving modes (Fig. 5, Table 4). As the travel speed increases, spatial distribution of high-accessibility areas becomes even. The average values of accessibility under the three transport modes all reach the 2025 target of Guangdong Province ( $2.6 \mathrm{~m}^{2}$ per capita), while only under driving mode is the 2025 target of Dongguan City met ( $3.2 \mathrm{~m}^{2}$ per capita).

Nonetheless, there are subtle differences between the results of the two methods. Overall, the spatial differentiation of accessibility measured by the MG2SFCA is more prominent. Specifically, in areas with high accessibility, the overall accessibility calculated by MG2SFCA

19 is much higher than that calculated by Gaussian 2SFCA, whereas in areas with low accessibility, the overall accessibility calculated by MG2SFCA is generally lower than that calculated by

21 Gaussian 2SFCA. This indicates that the preferences of different age groups for sports facilities do exert an impact on the evaluation of spatial accessibility to some extent. Not considering 
1 sports preferences underestimates the inequality of accessibility and thus mismatches the real

2 situation.

3

5

6

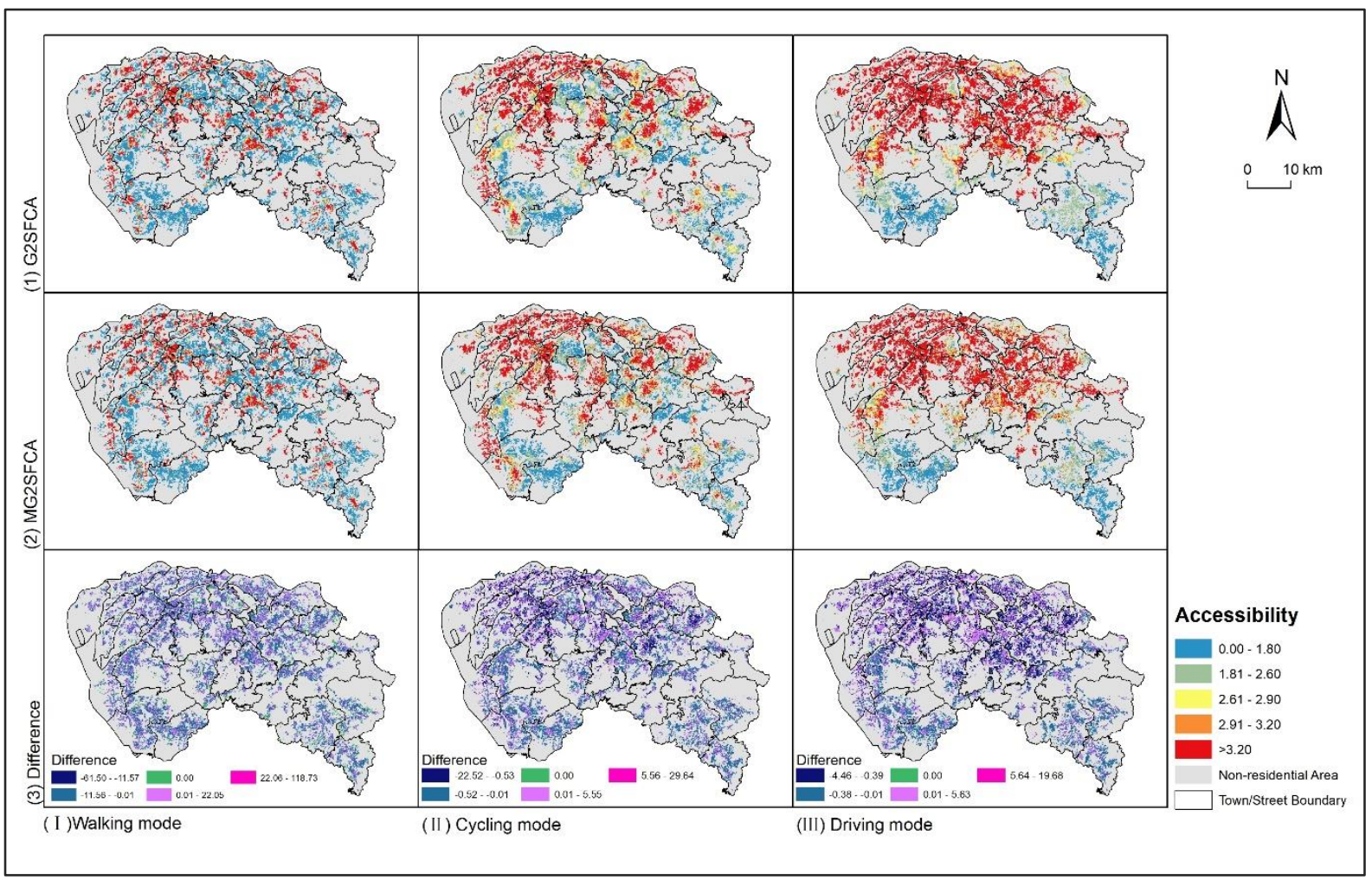

Figure 5. Spatial accessibility of sports facilities for total population

Table 4. The statistical characteristics of accessibility measured by MG2SFCA and Gaussian 2SFCA

MG2SFCA

Accessibility score
Gaussian 2SFCA

Walking mode Cycling mode Driving mode Walking mode Cycling mode Driving mode

\begin{tabular}{|c|c|c|c|c|c|c|}
\hline Median value & 1.32 & 2.67 & 3.46 & 1.28 & 2.58 & 3.26 \\
\hline Maximum value & 165.47 & 128.88 & 21.16 & 185.80 & 128.88 & 26.33 \\
\hline $0.00-1.80$ (Proportion) & $58.21 \%$ & $25.72 \%$ & $17.86 \%$ & $59.00 \%$ & $28.15 \%$ & $19.34 \%$ \\
\hline 1.81-2.60 (Proportion) & $9.98 \%$ & $22.40 \%$ & $13.91 \%$ & $10.04 \%$ & $22.37 \%$ & $14.94 \%$ \\
\hline
\end{tabular}




\begin{tabular}{ccccccc}
\hline $2.61-2.90$ (Proportion) & $3.09 \%$ & $7.80 \%$ & $4.82 \%$ & $2.96 \%$ & $6.88 \%$ & $6.47 \%$ \\
$2.91-3.20$ (Proportion) & $2.82 \%$ & $6.67 \%$ & $6.63 \%$ & $2.74 \%$ & $5.71 \%$ & $7.81 \%$ \\
$3.21-7.00$ (Proportion) & $17.63 \%$ & $34.08 \%$ & $56.18 \%$ & $16.82 \%$ & $32.27 \%$ & $47.80 \%$ \\
$>7.00$ (Proportion) & $8.27 \%$ & $3.33 \%$ & $0.61 \%$ & $8.44 \%$ & $4.62 \%$ & $3.64 \%$ \\
\hline
\end{tabular}

2 5. Discussion

3 5.1 Main findings

4 Generally, the evaluation results show significant disparities in spatial accessibility to sports

5 facilities among different regions in Dongguan. Defining the standard as $1.8 \mathrm{~m}^{2}$ per capita

6 stipulated in the 13th Five-Year Plan, the accessibilities measured by MG2SFCA are classified

7 as sufficient $(\geq 1.8)$ or insufficient $(<1.8)$ (Fig. 6$)$. Areas with adequate accessibility are mainly

8 distributed in the north, which can be attributed to the supply of facilities adapted to residents'

9 needs and well-developed transport networks. Regardless of the transport mode used, the

10 spatial accessibility of sports facilities in the southwest and southeast is at a relative

11 disadvantage. The reasons for underservice varied in these areas. Although Chang'an and

12 Humen Town in the southwest have a large number of sports facilities, they received lower

13 accessibility scores due to the large population. Conversely, towns and subdistricts in the

14 southeast with fewer sports facilities but smaller populations received higher scores. 


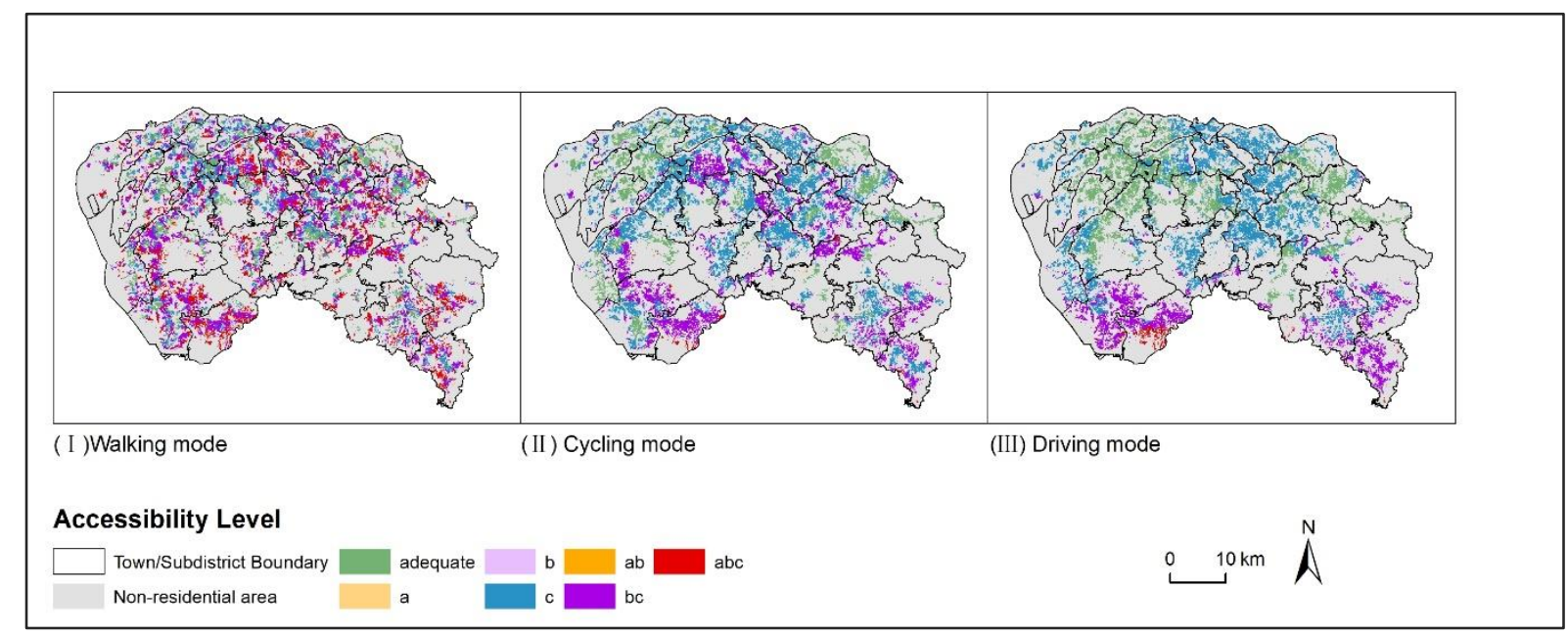
incorporated into the catchment and the overall supply of facilities becoming more balanced. 17 In our analysis, we assumed that all age groups equally chose walking, cycling, and driving as

Figure 6. Overall spatial accessibility level of sports facilities

Notes: 'a', 'b', 'c' represent insufficient accessibility of people aged 0-14, 15-59, and 60 years and above, respectively. For example, 'ab' indicates insufficient accessibility in both children and working-age groups.

Despite a similar spatial distribution pattern among the three age groups, the spatial accessibility scores are not equitable. Regardless of the mode of transport used, spatial accessibility continues to decline with increasing age. Nonetheless, among the three groups, the spatial differentiation of access to sports facilities in the children group was the most prominent, indicating that the supply of sports services suitable for children in Dongguan is adequate but relatively inequitable.

In addition, the use of different travel modes generated distinct accessibility results. As travel modes shifted from walking to cycling and driving, travel ranges expanded (i.e. distance thresholds increase), thereby leading to facilities inaccessible in walking mode being 
1 modes of transport. However, in reality, children and the elderly may be more inclined to walk,

2 reducing accessibility to sports facilities than our model suggests. Moreover, the fluctuation of

3 the maximum values of MG2SFCA scores under multiple transport modes reveals that it is

4 more reasonable to introduce the driving mode into spatial accessibility analysis.

6 Methodologically, our results confirm that the application of different methods for accessibility

7 measurement can influence the results (Apparicio et al., 2017). In this case, the MG2SFCA

8 method offers an insight into how preferences of different age groups might affect the real

9 accessibility of sports facilities. When the Gaussian 2SFCA is applied, the spatial inequality of

10 accessibility is weakened by the ignorance of preferences, which deviates from the actual

11 situation due to inclusion of facilities not commonly used by a specific population in the

12 analysis. For example, a senior citizen may be less likely to use a nearby basketball court than

13 a young adult who lives away from the same court. Thus, it is necessary to consider the

14 demographic and socioeconomic characteristics of individuals when measuring spatial

15 accessibility to better meet demands for refined services.

\section{$17 \quad 5.2$ Strengths and limitations}

18 Compared with the Gaussian 2SFCA metric, the proposed method has several strengths. The

19 use of individual-based population data allowed us to divide the total population into different

20 age groups; thus, age, an important factor affecting destination choice, can be included in the

21 accessibility analysis. In addition, the high-precision $100 \mathrm{~m} \times 100 \mathrm{~m}$ gridded population data

22 provided more accurate information on the number and geographic location of demanders, 
1 thereby delineating a more realistic spatial accessibility. One of the most obvious strengths is

2 that, rather than assuming that facilities can serve all people within a catchment, we identified

3 which facilities might serve which groups of people with the introduction of preference

4 heterogeneity indexes, which are calculated based on the use of each facility type by the total

5 population derived from a large national sampling survey (i.e. CHNS 2015). Then, the

6 preference heterogeneity index is converted into a weight of service delivery in combination

7 with the number of people, thus giving an idea of what percentage of the facility is likely to be

8 used by a certain age group. The MG2SFCA method allocates variable travel time thresholds

9 for different levels of sports facilities according to their service scope, thus accurately

10 identifying underserved areas. Moreover, the proposed model examines and compares spatial

11 accessibility to facilities through three common modes of transport (walking, cycling, and

12 driving), which is a supplement to the previous measures using only a single travel mode

13 (usually the driving mode). This is because the introduction of multiple travel modes identified

14 significant discrepancies in accessibility and reaffirmed a previous finding that a larger travel

15 time threshold improves spatial accessibility (Luo and Wang, 2003).

17 However, several limitations should be noted. First, the MG2SFCA method only reflects the spatial accessibility to sports facilities based on different ages, ignoring other non-spatial

19 factors (e.g. demographic profiles and socioeconomic status). While age is an important determinant of facility preference, indicators such as personal interests, household income and car ownership may exert a stronger influence on facility selection. From a service point of view,

22 the capacity, quality, supporting activities, availability, affordability, ancillary facilities (e.g. 
1 locker rooms, shower rooms, restaurants, and parking lots), and other characteristics of services

2 significantly influence people's perceptions of specific facilities. Second, we used running

3 speeds based on the road grade instead of actual commuting speeds, which may result in biased

4 accessibility scores. In addition, we only considered three independent travel modes, not

5 considering travelling by public transit or a combination of multiple transport modes, which

6 occurs especially in metropolitan cities. Third, the thresholds for different levels of sports

7 facilities are ideal values set according to service radius, rather than the coverage that facilities

8 can actually provide and the travel time that people are willing to spend. Fourth, we did not

9 distinguish between fee-paying and free services, nor between public and private services.

10 Finally, outputs from the MG2SFCA models are relative instead of absolute, which can only

11 be employed for comparing spatial accessibility between smaller geographical units in the 12 study area.

14 In view of the limitations discussed above, future research on accessibility measurements

15 should be conducted in several directions. First, in addition to spatial accessibility, other crucial

16 factors that play an important role in the decision-making process, such as information access,

17 economic access, socio-cultural access, and social-managerial environment should also be considered (Cheng et al., 2012). In this regard, it is recommended to supplement individual

19 attributes such as gender, education, work, economic conditions, acceptable travel time, payment amount and subjective preferences by means of surveys to systematically investigate the potential needs of different groups of people for services and reasonably determine the appropriate size of the catchment. Field studies are also required to gather information on the 
1 services' openness (i.e. which citizens are allowed), pricing, comfort level, and technical

2 features. In addition, future studies can obtain the actual travel distance and time between

3 providers and consumers through real-time traffic data provided by an online map platform,

4 which is conducive to the accurate evaluation of accessibility. Given that people do not always

5 travel to destinations, workplaces are suggested to be regarded as demand points to search for

6 available services within a catchment. Moreover, integrating multiple types of complex travel

7 behaviours into the analysis is helpful in improving the evaluation framework. More research

8 focusing on the actual usage rates of facilities is needed to accurately determine accessibility.

$10 \quad 5.3$ Policy suggestions

11 The findings of our study provide policymakers and planners with profound implications

12 concerning the spatial patterns of existing sports facilities and thus make improvements. To

13 improve the accessibility of sports facilities, the priority is to strengthen the development of

14 sporting infrastructure, including reducing the number of facilities in areas with excessive

15 provision and increasing the number of facilities in areas with poor service. In this regard, the

16 densely populated southwest region in Dongguan should appropriately expand the area of

17 sports facilities and increase the number of comprehensive venues to improve efficiency of

18 land use, while the sparsely populated southeast region should lay out more and varied sports

19 facilities near the residential neighbourhood, especially municipal facilities. It is also important

20 to reinforce facility maintenance, improve the venue environment, and provide ideal supporting

21 facilities to increase their attractiveness. Meanwhile, the types of facilities should be adjusted according to regional population attributes (i.e. age, sex, income, health and socio-cultural 
1 background) related to sporting preferences. Specifically, the construction of sports

2 infrastructure for middle-and low-intensity sports such as walking and table tennis, which are

3 mostly used by the elderly, should be done in Dongguan. In addition, the performance of

4 transport networks is crucial for spatial accessibility that urban planners should pay more

5 attention to while transport planning and road network designing to reduce traffic congestion.

6 Among them, the configuration of sidewalks and cycle paths suitable for short-distance travel

7 and public transport suitable for long-distance travel is particularly critical. The results of our

8 study also imply that the indicators used to guide the construction of sports facilities in the

9 current standards may be overgeneralised. Per capita area cannot be regarded as a 'one-size-

10 fits-all' standard. Thus, the government may need to set relevant standards according to the

11 proportion of the population in different demographic and socioeconomic groups within the

12 city.

\section{Conclusion}

15 This paper has suggested an improved model, namely the MG2SFCA method, based on the traditional 2SFCA for determining spatial accessibility. The proposed method not only considers three transport modes separately, but also classifies the total population by age and incorporates the weighting value of each age group's preference for different types of sports

19 facilities. Using comprehensive data and individual-based actual population data provided by the 'One standard and Three actualities' information database, we have applied a new method to estimate spatial patterns of accessibility to sports facilities among children, working-age adults, and the elderly under walking, cycling, and driving modes in Dongguan, China. Overall, 
1 the spatial layout of sports facilities roughly matches the distribution of the total population.

2 However, the results indicate an enormous disparity in spatial accessibility among different age

3 groups, transport modes, and regions in Dongguan. Specifically, the MG2SFCA score in the

4 north was generally higher than that in the south. Compared with cycling and driving modes,

5 the spatial accessibility in the walking mode presents a more striking distinction, which is

6 partially attributable to the restrictions in travel range. Across multiple age groups, regardless

7 of the travel mode used, children had the highest accessibility score, followed by the working-

8 age population, and finally, the elderly population.

10 The uneven distribution of sports facilities may have a negative impact on health. The

11 MG2SFCA method allows a more accurate and realistic spatial accessibility assessment,

12 making it possible to distinguish sufficiently served and underserved areas, thus contributing

13 to rational allocation and utilisation of public resources and a significant minimisation of

14 resource waste and inequity. To improve spatial accessibility, great improvements are needed

15 in terms of services (including location, level, size, function, type, quality, openness, and

16 affordability of facilities) and transport systems (including road network design, public

17 transport, and non-public transport). Furthermore, it is suggested that the public should be encouraged to participate in the planning and construction of sports infrastructure. Government

19 departments should also formulate relevant policies to stimulate physical activity to promote mental and physical health, with special attention to the needs of the older population. 


\section{Declarations}

\section{Ethics approval and consent to participate}

3 Not applicable.

4

\section{Consent for publication}

$6 \quad$ Not applicable.

7

$8 \quad$ Availability of data and materials

9 Data sharing is not applicable to this article as no datasets were generated or analysed during the current

10 study.

11

12 Competing interests

13 The authors declare that they have no competing interests.

$15 \quad$ Funding

16 This work was supported by the National Natural Science Foundation of China (Grant Numbers

1741871140 and 41971194), and the Fundamental Research Funds for the Central Universities (Grant

18 Numbers 20lgzd10).

19

20 Authors' contributions

21 TX and YL developed the research idea and completed the study design. TX conducted basic processing on the real population data. TD and XZ conducted basic processing on the data of sports facilities. TX, 
1 TD and XZ carried out the statistical analysis and wrote the manuscript. TX, YL and ZT revised the

2 manuscript. All authors read and approved the final manuscript.

\section{Acknowledgements}

$5 \quad$ Not applicable.

7 Authors' information

$8{ }^{1}$ School of Geography and Planning, Sun Yat-Sen University, Xingang Xi Road, Guangzhou 510275,

9 China. ${ }^{2}$ Guangdong Key Laboratory for Urbanization and Geo-simulation, Sun Yat-Sen University,

10 Xingang Xi Road, Guangzhou 510275, China. ${ }^{3}$ Faculty of Geographical Science, Beijing Normal 11 University, No.19, XinJieKouWai St., Haidian District, Beijing 100875, China.

\section{References}

14 Apparicio, P., Gelb, J., Dubé, A., Kingham, S., Gauvin, L., Robitaille, É., 2017. The approaches to measuring the potential spatial access to urban health services revisited: distance types and aggregation-error issues. Int. J. Health Geogr. 16, 32.

Bélanger, M., Townsend, N., Foster, C., 2011. Age-related differences in physical activity profiles of English adults. Prev. Med. 52, 247-249.

Bell, S., Wilson, K., Bissonnette, L., Shah, T., 2013. Access to Primary Health Care: Does Neighborhood of Residence Matter? Ann. Assoc. Am. Geogr. 103 (1), 85-105. Weber, C., Chaix, B., 2011. Investigating disparities in spatial accessibility to and 

Place 17 (1), 114-121.

3 Bourke, M., Hilland, T. A., Craike, M., 2021. A systematic review of the within-person association between physical activity and affect in children's and adolescents' daily lives. Psychol. Sport Exerc. 52, 101825.

6 Cereijo, L., Gullón, P., Cebrecos, A., Bilal, U., Santacruz, J. A., Badland, H., Franco, M., 2019. Access to and availability of exercise facilities in Madrid: an equity perspective. Int. J. Health Geogr. 18,15 .

Chen, B. Y., Cheng, X.-P., Kwan, M.-P., Schwanen, T., 2020a. Evaluating spatial accessibility to healthcare services under travel time uncertainty: A reliability-based floating catchment area approach. J. Transp. Geogr. 87, 102794.

12 Chen, Z., Zhou, X., Yeh, A. G. O., 2020b. Spatial accessibility to kindergartens using a spectrum combinational approach: Case study of Shanghai using cellphone data. Environ. Plan. B: Urban.

Cheng, Y., Rosenberg, M. W., Wang, W., Yang, L., Li, H., 2012. Access to residential care in Beijing, China: making the decision to relocate to a residential care facility. Ageing Soc. 32, 1277-1299.

Cutumisu, N., Spence, J. C., 2012. Sport Fields as Potential Catalysts for Physical Activity in the Neighbourhood. Int. J. Environ. Res. Public Health 9, 294-314.

Dai, D., 2010. Black residential segregation, disparities in spatial access to health care facilities, and late-stage breast cancer diagnosis in metropolitan Detroit. Health Place 16, 1038-1052.

Dai, D., 2011. Racial/ethnic and socioeconomic disparities in urban green space accessibility: Where to intervene? Landscape Urban Plan. 102, 234- 244.

Dai, D., Wang, F., 2011. Geographic disparities in accessibility to food stores in southwest Mississippi. 
2 Delamater, P. L., 2013. Spatial accessibility in suboptimally configured health care systems: A modified two-step floating catchment area (M2SFCA) metric. Health Place 24, 30-43.

Dongguan Bureau of Statistics, National Bureau of Statistics of China, 2020. Dongguan statistical yearbook 2020. Beijing: China Statistical Press (In Chinese).

Dongguan Municipal People's Government, 2020. Circular of Dongguan Municipal People's Government Office on Printing and Distributing Implementation Opinions on Optimizing the Allocation of Public Service Resources. Bulletin of Dongguan Municipal People’s Government. (7), 10-15 (In Chinese).

Farrell, L., Shields, M. A., 2002. Investigating the economic and demographic determinants of sporting participation in England. J. R. Statist. Soc. 165 (2), 335-348.

Fransen, K., Neutens, T., Maeyer, P. D., Deruyter, G., 2015. A commuter-based two-step floating catchment area method for measuring spatial accessibility of daycare centers. Health Place 32, $65-73$.

Gyasi, R. M., Phillips, D. R., Asante, F., Boateng, S., 2021. Physical activity and predictors of loneliness in community-dwelling older adults: The role of social connectedness. Geriatr. Nurs. 42 (2), $592-598$.

Halonen, J. I., Stenholm, S., Kivimäki, M., Pentti, J., Subramanian, S. V., Kawachi, I., Vahtera, J., 2015. Is change in availability of sports facilities associated with change in physical activity? A prospective cohort study. Prev. Med. 73, 10-14.

Hansen, W. G., 1959. How Accessibility Shapes Land Use. J. Am. Inst. Plann. 25 (2), 73-76.

Heath, G. W., Parra, D. C., Sarmiento, O. L., Andersen, L. B., Owen, N., Goenka, S., Montes, F., 
Brownson, R. C., 2012. Evidence-based intervention in physical activity: lessons from around the world. The Lancet. 380 (9838), 272-281.

Higgs, G., Langford, M., Norman, P., 2015. Accessibility to sport facilities in Wales: A GIS-based analysis of socioeconomic variations in provision. Geoforum 62, 105-120.

Hu, L., Zhao, C., Wang, M., Su, S., Weng, M., Wang, W., 2020. Dynamic healthy food accessibility in a rapidly urbanizing metropolitan area: Socioeconomic inequality and relative contribution of local factors. Cities 105, 102819.

Humpel, N., Owen, N., Leslie, E., 2002. Environmental Factors Associated with Adults' Participation in Physical Activity: A review. Am. J. Prev. Med. 22 (3), 188-199.

Hunter, D. J., Reddy, K. S., 2013. Noncommunicable Diseases. N. Engl. J. Med. 369 (14), 1336-1343.

Jamtsho, S., Corner, R., Dewan, A., 2015. Spatio-Temporal Analysis of Spatial Accessibility to Primary Health Care in Bhutan. ISPRS Int. J. Geoinf. 4, 1584-1604.

Karusisi, N., Thomas, F., Méline, J., Chaix, B., 2013. Spatial accessibility to specific sport facilities and corresponding sport practice: the RECORD Study. Int. J. Behav. Nutr. Phys. Act. 10, 48.

Kwan, M. P., 1998. Space-Time and Integral Measures of Individual Accessibility: A Comparative Anal ysis Using a Point-based Framework. Geogr. Anal. 30 (3), 191-216.

Langford, M., Higgs, G., Fry, R., 2016. Multi-modal two-step floating catchment area analysis of primary health care accessibility. Health Place 38, 70-81.

Langford, M., Higgs, G., Radcliffe, J., 2018. The application of network-based GIS tools to investigate spatial variations in the provision of sporting facilities. Ann. Leis. Res. 21 (2), 178-198.

Li, Z., Wei, H., Wu, Y., Su, S., Wang, W., Qu, C., 2019. Impact of community deprivation on urban park access over time: Understanding the relative role of contributors for urban planning. Habitat 
Int. 92, 102031.

2 Lin, Y., Wan, N., Sheets, S., Gong, X., Davies, A., 2018. A multi-modal relative spatial access assessment approach to measure spatial accessibility to primary care providers. Int. J. Health Geogr. 17, 33.

Liu, Y., Wang, X., Zhou, S., Wu, W., 2019. The association between spatial access to physical activity facilities within home and workplace neighborhoods and time spent on physical activities: evidence from Guangzhou, China. Int. J. Health Geogr. 19, 22.

Luo, J., 2014. Integrating the Huff Model and Floating Catchment Area Methods to Analyze Spatial Access to Healthcare Services. Trans. GIS 18 (3), 436-448.

Luo, W., Qi, Y., 2009. An enhanced two-step floating catchment area (E2SFCA) method for measuring spatial accessibility to primary care physicians. Health Place 15, 1100-1107.

Luo, W., Wang, F., 2003. Measures of spatial accessibility to health care in a GIS environment: synthesis and a case study in the Chicago region. Environ. Plann. B: Plann. Des. 30, 865-884.

Luo, W., Whippo, T., 2012. Variable catchment sizes for the two-step floating catchment area (2SFCA) method. Health Place 18, 789-795.

Mao, L., Nekorchuk, D., 2013. Measuring spatial accessibility to healthcare for populations with multiple transportation modes. Health Place 24, 115-122.

McGrail, M. R., Humphreys, J. S., 2014. Measuring spatial accessibility to primary health care services: Utilising dynamic catchment sizes. Appl. Geogr. 54, 182-188.

Morris, J. M., Dumble, P. L., Wigan, M. R., 1979. Accessibility Indicators for Transport Planning. Transpn. Res. A 13A, 91-109.

National Bureau of Statistics of China, 2019. Statistical Communique of The People's Republic of 

(In Chinese).

3 Neutens, T., 2015. Accessibility, equity and health care: review and research directions for transport geographers. J. Transp. Geogr. 43, 14-27.

Ngui, A. N., Apparicio, P., 2011. Optimizing the two-step floating catchment area method for measuring spatial accessibility to medical clinics in Montreal. BMC Health Serv. Res. 11 (166).

7 People's Government of Guangdong Province, 2020. Circular of the People's Government of Guangdong Province on Printing and Distributing the Implementation Outline of Building a Strong Sports Province in Guangdong Province. Bulletin of the People's Government of Guangdong Province. (26), 2-10 (In Chinese).

Pirie, G. H., 1979. Measuring Accessibility: A Review and Proposal. Environ. Plann. A 11 (3), 299-312.

Popkin, B. M., Du, S., Zhai, F., Zhang, B., 2010. Cohort Profile: The China Health and Nutrition Survey - Monitoring and understanding socio-economic and health change in China, 1989-2011. Int. J. Epidemiol. 39 (6), 1435-1440.

Radke, J., Mu, L., 2000. Spatial Decompositions, Modeling and Mapping Service Regions to Predict Access to Social Programs. Geographic Information Sciences 6 (2), 105-112.

Senaratne, N., Stubbs, B., Werneck, A. O., Stamatakis, E., Hamer, M., 2021. Device-measured physical activity and sedentary behaviour in relation to mental wellbeing: An analysis of the 1970 British cohort study. Prev. Med. 145.

Shrestha, S., Kestens, Y., Thomas, F., Aarbaoui, T. E., Chaix, B., 2019. Spatial access to sport facilities from the multiple places visited and sport practice: Assessing and correcting biases related to selective daily mobility. Soc. Sci. Med. 236, 112406. 
1 Subal, J., Paal, P., Krisp, J. M., 2021. Quantifying spatial accessibility of general practitioners by applying a modified huff three-step floating catchment area (MH3SFCA) method. Int. J. Health Geogr. 20, 9 .

Tao, Z., Cheng, Y., 2016. Research progress of the two-step floating catchment area method and extensions. Prog. Geogr. 35 (5), 589-599 (In Chinese)..

Tao, Z., Cheng, Y., Dai, T., 2014. Measuring spatial accessibility to residential care facilities in Beijing. Prog. Geogr. 33 (5), 616-624 (In Chinese).

Tao, Z., Cheng, Y., Du, S., Feng, L., Wang, S., 2020a. Accessibility to delivery care in Hubei Province, China. Soc. Sci. Med. 260, 113186.

Tao, Z., Cheng, Y., Liu, J., 2020b. Hierarchical two-step floating catchment area (2SFCA) method: 146. 
1 White, R. L., Babic, M. J., Parke, P. D., Lubans, D. R., Astell-Burt, T., Lonsdale, C., 2017. DomainSpecific Physical Activity and Mental Health: A Meta-analysis. Am. J. Prev. Med. 52 (5), 653666.

World Health Organization (WHO), Global action plan on physical activity 2018-2030: more active people for a healthier world. World Health Organization, Geneva, 2018.

6 World Health Organization (WHO). Global Physical Activity Questionnaire (GPAQ) Analysis Guide. Available at: https://www.who.int/ncds/surveillance/steps/GPAQ/en/ (accessed 10 February 2020).

Xia, T., Song, X., Zhang, H., Song, X., Kanasugi, H., Shibasaki, R., 2019. Measuring spatio-temporal

Xing, L., Liu, Y., Liu, X., 2018. Measuring spatial disparity in accessibility with a multi-mode method based on park green spaces classification in Wuhan, China. Appl. Geogr. 94, 251-261.

13 Yang, D.-H., Goerge, R., Mullner, R., 2006. Comparing GIS-Based Methods of Measuring Spatial Accessibility to Health Services. J. Med. Syst. 30 (1), 23-32. recreational and non-recreational physical activity with mental health: A Canadian crosssectional analysis. JAD Reports 1, 100021. 


\section{Supplementary Files}

This is a list of supplementary files associated with this preprint. Click to download.

- Highlights.docx 